\title{
The Microstructure of Unirradiated and Neutron Irradiated Inconel X750
}

\author{
O.T. Woo*, C.D. Judge*, H.M. Nordin*, D. Finlayson*, and C. Andrei** \\ * AECL, Chalk River Laboratories, Chalk River, ON K0J 1P0, CANADA \\ ** Canadian Centre for Electron Microscopy, McMaster University, Hamilton, CANADA
}

Inconel X750 is a high temperature, age-hardenable superalloy that has excellent corrosion properties and good mechanical strength and ductility, making it suitable for use in nuclear reactors [1]. These physical properties can change during reactor operation as neutrons cause displacement damage by knocking atoms out of their lattice sites, producing dislocation loops, tangles, voids and precipitates $[1,2]$. As a first step to understand the effect of neutron irradiation, unirradiated and irradiated X750 were characterized by X-ray diffraction (XRD), light and electron (SEM, TEM) microscopy. X750 was solution treated for $<15$ minutes, cold-worked and then age-hardened at a temperature of 700 to $750^{\circ} \mathrm{C}$ for $16 \mathrm{~h}$. Analyses of the material indicated major chemical constituents (in wt.\%) as follows: Ni, 74.0; Cr, 15.0; Fe, 7.30; Ti, 2.50; Al, 0.68; Nb, 0.99; Mn, 0.15; C, 0.067.

The microstructure of the heat-treated X750 consists of equiaxed grains of the fcc $\gamma$-matrix (Figure 1), with many $\{111\}$ deformation twins, and large $\mu \mathrm{m}$ size blocky or cuboidal inclusions notably those aligned to form stringers (see Figure 2); smaller precipitates were seen at the grain boundaries and in the grain interior. The mean grain size was $(12 \pm 8) \mu \mathrm{m}$ in diameter, with a maximum of $45 \mu \mathrm{m}$. The large inclusions were readily analyzed by energy dispersive X-ray (EDX) analysis to be rich in Ti and $\mathrm{Nb}$, and electron diffraction and XRD confirmed that they were fcc $(\mathrm{Ti}, \mathrm{Nb}) \mathrm{C}$, the $\mathrm{MC}$ carbide. The small precipitates seen in Figure 1 were predominantly fcc $\mathrm{M}_{23} \mathrm{C}_{6}$ carbides that were a few tenths of $1 \mu \mathrm{m}$ in diameter, and contained $\approx 90 \mathrm{wt} \% \mathrm{Cr}$. Ordered fcc $\gamma^{\prime}\left(\mathrm{Ni}_{3} \mathrm{Al}\right)$ precipitates have a lattice parameter that differ from $\gamma$ phase by about $0.5 \%$, these precipitates averaged about $15 \mathrm{~nm}$, but less than $25 \mathrm{~nm}$ in diameter, and they were difficult to find in the TEM foils. Using the contrast from $\{110\}$ and $\{100\}$ superlattice diffraction spots to image the precipitates it was observed that they were distributed non-uniformly within the grains and they were not found in every $\gamma$-grain. The absence of shoulders in the main $\gamma$-phase peaks using X-ray diffraction suggests that their volume fraction is small $(<1 \%)$. HCP Ni 3 Ti $\eta$-phase was present as rod-shaped precipitates across the $\gamma$-matrix grains. They had an orientation relationship with the matrix of $(111)_{\gamma} / /(0001)_{\eta}$, and $\left[\begin{array}{ll}1 & 0\end{array}\right]_{\gamma} / /\left[\begin{array}{ll}11 & 0\end{array}\right]_{\eta}$. Figures 3 and 4 are examples of these phases in the unirradiated X750.

Apart from radiation-induced dislocation loops and precipitates, TEM examination of foils made from neutron-irradiated X750 revealed the presence of cavities less than $5 \mathrm{~nm}$ in size. Figures 5(a) and (b) were recorded in the under- and over-focussed conditions showing Fresnel contrasts, where the cavities appeared bright with a dark fringe at under-focus, but became dark with a bright fringe at over-focussed condition. These cavities were shown to be within the foil by taking stereopairs on the TEM and examining them in a stereo-viewer. No such cavities were seen in the unirradiated X750. Thermal neutrons create radiation damage in Ni by capture, causing transmutation of the nucleus, followed by particle emission. The recoil of the transmutated nucleus and the emission of protons and alpha particles from the nuclear reactions lead to displacement damage and the creation of gas atoms in the material. Thus the cavities observed here can be vacancy rich regions or gas bubbles or a combination of both. Further work is required to study the damage structure.

[1] W.J. Mills et al., Nuclear Technology 73 (1986) 102.

[2] T.R. Allen et al., J. Nuclear Materials 270 (1999) 290. 

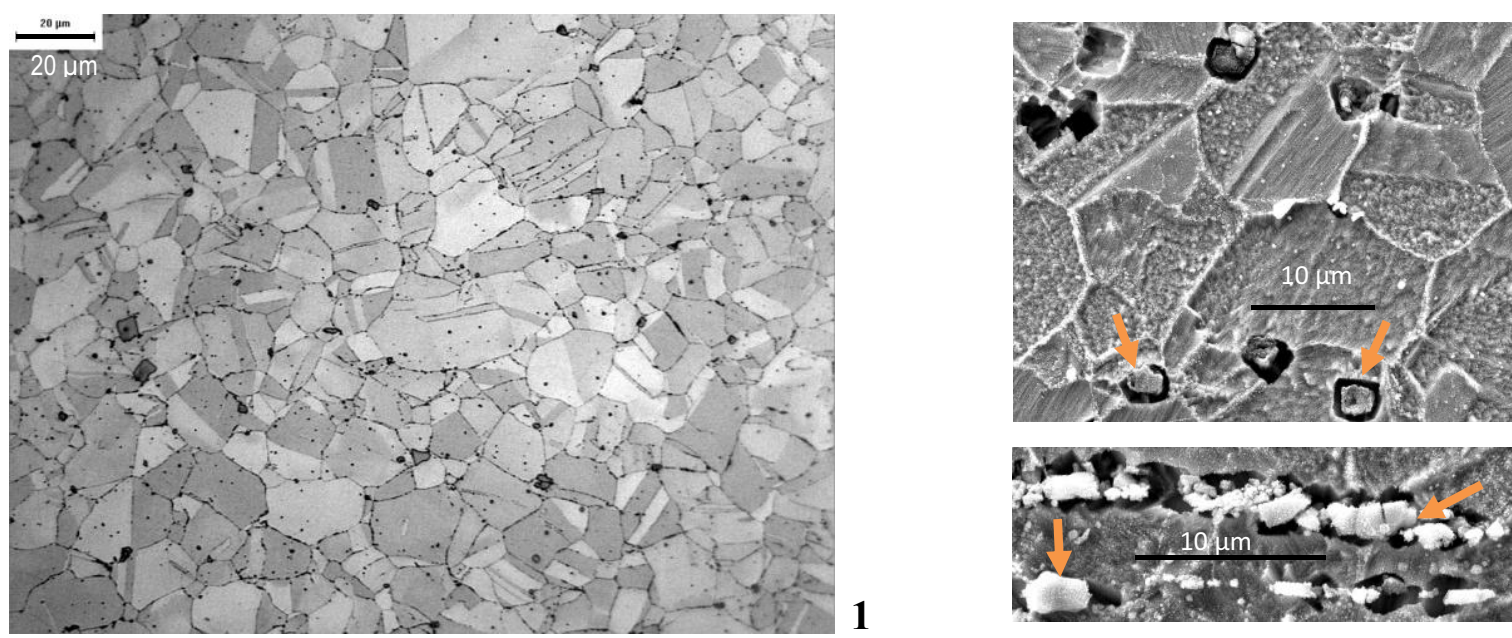

2(a)

1
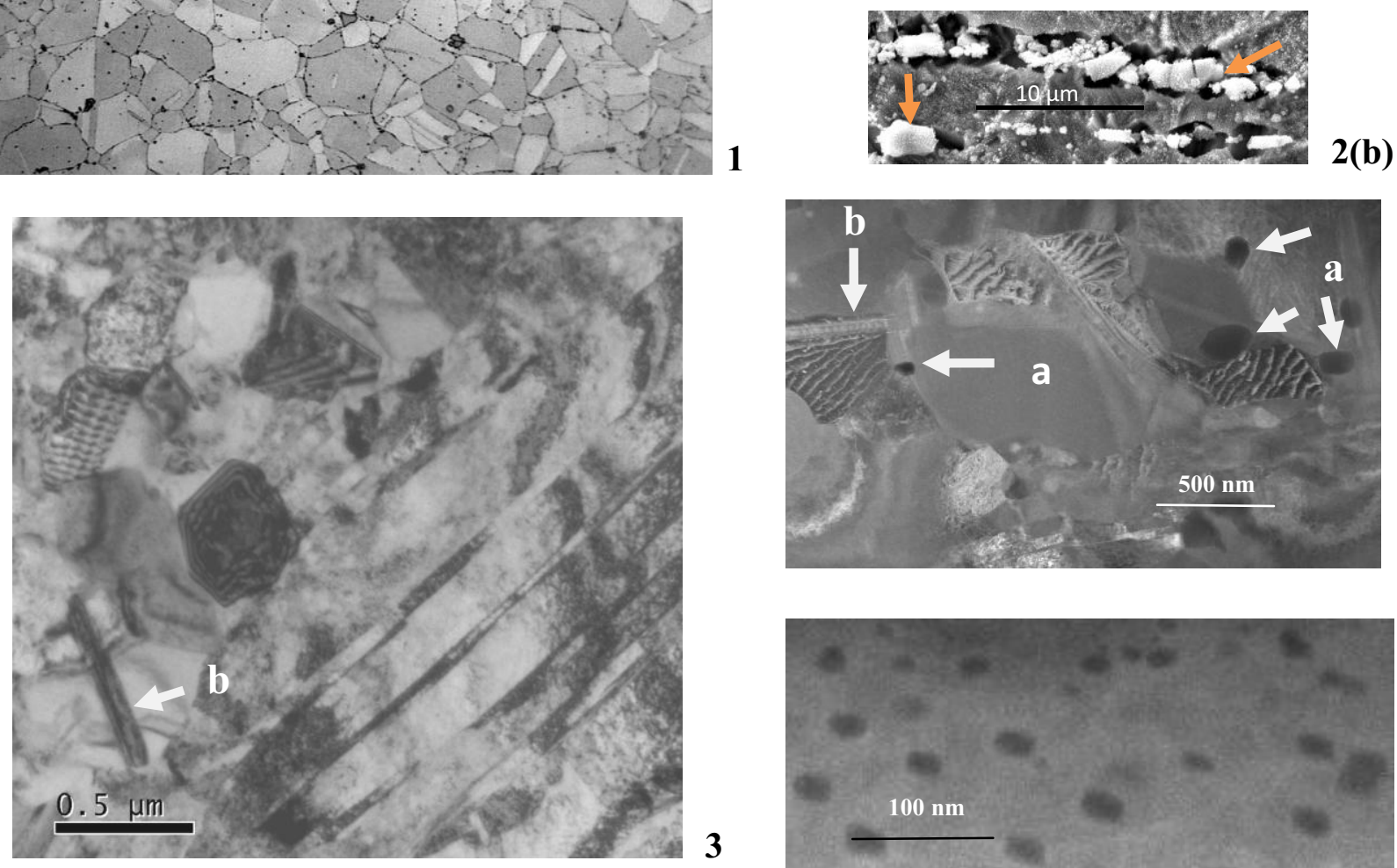

4(a)

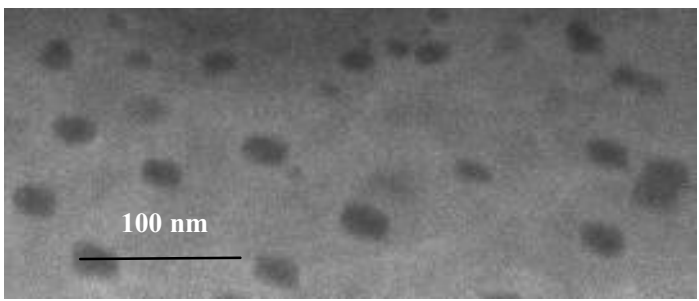

4(b)

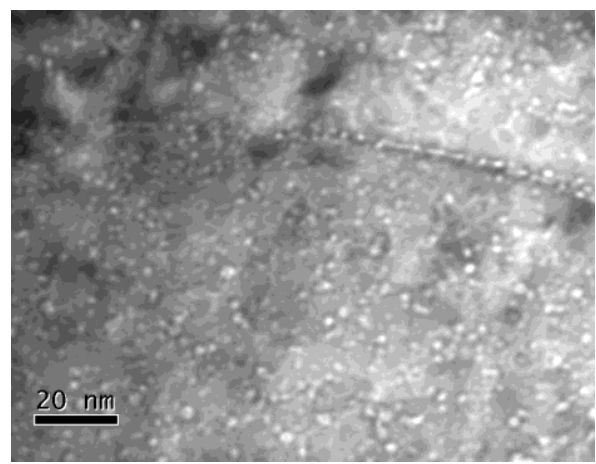

5(a)

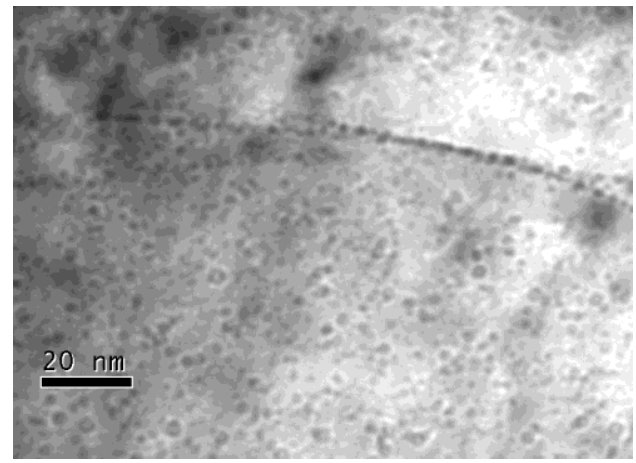

5(b)

Figure 1. Optical Micrograph of X750 showing equiaxed grains with precipitates and twins.

Figure 2. Blocky or cuboidal inclusions in X750, or at stringers, indicated by arrows.

Figures $3 \& 4$. Phases indicated by: a, $\mathrm{M}_{23} \mathrm{C}_{6}$ carbide, $b$, $\eta$-phase. $\gamma^{\prime}$ particles shown in $4(\mathrm{~b})$ are $\leq 25 \mathrm{~nm}$ in diameter. Figures 5(a) and (b) show $<5 \mathrm{~nm}$ diameter cavities, at under-focus in (a) and over-focus in (b). 